\title{
Attentional bias towards high and low caloric food on repeated visual food stimuli: An ERP study
}

\author{
Aruna Duraisingam ${ }^{1}$, Ramaswamy Palaniappan ${ }^{1}$ and Daniele Soria ${ }^{1}$
}

\begin{abstract}
Food variety influences appetitive behaviour, motivation to eat and energy intake. Research found that repeated exposure to varied food images increases the motivation towards food in adults and children. This study investigates the effects of repetition on the modulation of early and late components of event-related potentials (ERPs) when participants passively viewed the same food and non-food images repeatedly. The motivational attention to food and non-food images were assessed in frontal, centroparietal, parietooccipital and occipitotemporal areas of the brain. Participants showed increased late positive potential (late ERP component) to high caloric image in the occipitotemporal region compared to low caloric and nonfood images. Similar effects could be seen in the early ERP component in the frontal region, but with reversed polarity. Data suggest that both the early and late ERP components show greater ERP amplitude when viewing high caloric images than low caloric and non-food images. Despite repeated exposure to same image, high caloric food continued to show sustained attention compared to low caloric and non-food image.
\end{abstract}

\section{INTRODUCTION}

Obesity is increasing throughout the world, causing longterm health conditions such as type- 2 diabetes, cardiovascular disease, etc. [1]. Obesity can be interpreted as the lack of ability to adjust food intake for maintaining a good balance of food and energy consumption. This poor nutrition intake behaviour is mainly guided by pre-ingestion choices driven by visual food appearance, mainly through repeated food exposure. Repeated food exposure has been used as a key marketing and advertisement strategy by many multinational chains in the food industry [2].

In obesity-related research, developing effective treatment and intervention techniques are challenging, and it is crucial to gain more insight into the mechanism of regulation in response to food stimuli. Previous research studies demonstrate that food images are readily differentiated according to the energy/caloric content, and related motivation behaviour modulates the amplitude of late positive potentials (LPPs) measured over the centroparietal brain region (around 250$500 \mathrm{~ms}$ ); larger LPPs can be viewed for high caloric food images than low caloric food and neutral images [3], [4]. Moreover, in the early ERP window $(\sim 150-200 \mathrm{~ms})$, the research found increased negative amplitude (N2) for chocolate images more than neutral image in binge eating individuals [4]. This differentiation in early ERP component and LPP modulation can be interpreted as individuals' motivational significance towards external stimuli that might be mediated by the activation of cortico-limbic appetitive and defensive

\footnotetext{
${ }^{1}$ All authors are with Data Science Research Group, School of Computing, University of Kent, Canterbury, UK A.Duraisingam/R.Palani/D.Soria@kent.ac.uk
}

systems, supporting individuals' perception and action [5] Research also suggests that these early and late alterations in behavioural and cognitive response patterns can be interpreted in terms of implicit memory processes [6].

Previous research on repeated food stimuli mainly investigated individuals' cognitive responses and motivational behaviour by presenting different food class images based on varied caloric value to participants (presenting via varied, i.e. inter-mixed images in each class) [3], [4]. Those research studies found that LPP's affective modulation appears less susceptible to repetition effects for high energy content food images than responses to low energy and neutral food images. This enhancement in LPPs could be caused by the influence of diverse food images (different presentation of the same food or different varieties of food) presented repeatedly, as appetite behaviour is strongly influenced by the variety of food [7], [8]. This differentiation in food variety might be highly related to food reward properties, initial cognitive encoding, and attentional allocation processes of different food images presented repeatedly [3], [4]. This opens the question of whether repeated exposure to the same visual food image presented repeatedly shows any difference in the modulation of the cognitive process for different energy value foods. However, this has not been investigated so far.

Therefore, this study investigates whether there is any differentiation in motivation behaviour and cognitive responses when the same food image is repeatedly presented (free viewing) and analysed in different brain regions. For this purpose, data were collected from participants when they passively viewed the same food (one high caloric image and one low caloric image) and non-food image repeatedly for 40 times each. The purpose of this study is to compare the behavioural responses to the repeated presentation of images using well developed EEG signal analysis techniques.

\section{METHODOLOGY}

\section{A. Participants}

Eleven participants (seven males and four females) were recruited via campus announcements. Participants' age ranged between 23 and 48 years (mean $=38.40 \pm 6.47$ ). Liking of apple and pizza were evaluated using a six-point Likert scale $(0=$ low; $5=$ high $)$, and only participants who scored above 2 for both pizza and apple were included. The main exclusion criterion was neurological or current mental disorders (e.g., eating disorders). Body mass index (BMI) ranged from 22.20 to $39.15 \mathrm{Kg} / \mathrm{m}^{2}$ (mean $=29.01 \pm 5.82$ ) with 7 participants (4 female and 3 male) being overweight or 


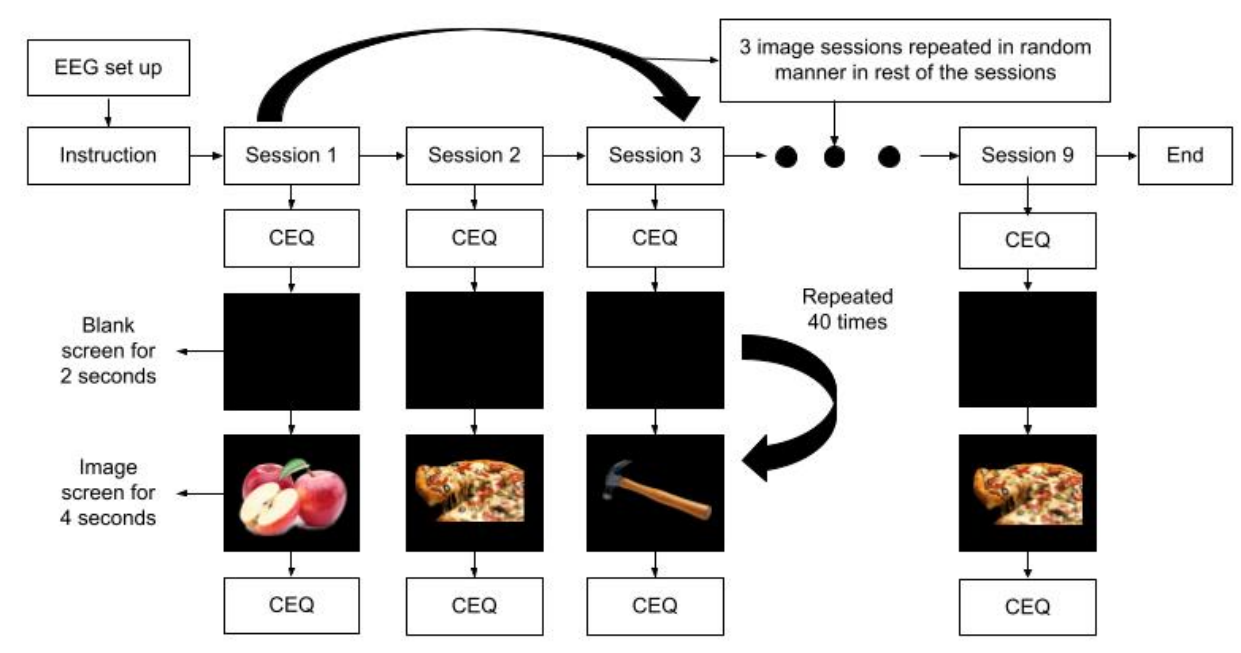

Fig. 1: Experimental design, EEG - Electroencephalogram, CEQ - Craving Experience Questionnaire (participants record their current craving intensity).

obese. The study was approved by the Faculty of Sciences Research Ethics Committee at the University of Kent.

\section{B. Experimental Design and Procedure}

Visual stimuli consisted of three images - apple, pizza (representing low and high caloric food respectively), and hammer as a non-food image - which participants viewed at a distance of one meter on a monitor screen. Each subject participated in nine sessions ( 3 images X 3 times) on the same day. In each session, one image (randomly chosen among either high caloric, low caloric or non-food) was presented for four seconds with two seconds of interstimulus interval, for a total of 40 times (i.e. 40 trials). At the start and end of each session, food craving intensity was measured using the Craving Experience Questionnaire (CEQ). Participants were allowed to take up to five minutes of break between each session. Fig. 1 shows a graphical description of the experimental paradigm.

Participants firstly provided written informed consent and completed a screening questionnaire to check for inclusion and exclusion criteria. Those who were eligible to participate were invited for EEG data collection. In order to limit the direct implication of the participants' hunger state on ERP responses and make it comparable, all participants were requested to have a good breakfast and were instructed to refrain from eating for at least three hours before data collection [9]. All experiments were conducted at 12 noon. After arriving at the lab, participants were asked to fill the Dutch Eating Behaviour Questionnaire, then EEG electrodes were placed. Participants were carefully briefed about the experiment (of which the visual design was developed using the Psych toolbox) and were given a test trial if requested. Subsequently, participants viewed one image in the rest of the sessions(each session lasting approximately five minutes) whilst EEG data were recorded.

\section{EEG Recording and Data Processing}

Data was recorded with Neuroelectric's StarStim 32 electrodes device placed according to the 10-10 system. EEG was recorded with a sampling rate of $500 \mathrm{~Hz}$. Two electrodes CMS-Common Mode and DRL-Driven Right Leg (collected via an earclip) were used as reference and ground. Matlab (R2020b), its plugin EEGLAB (v2020.0) and ERPLab (v8.10) were used for analysis. EEG data processing consisted of low pass filtering at $30 \mathrm{~Hz}$ (Butterworth IIR filter) and standardised PREP pre-processing pipeline [10] applied for line noise removal. The cleaned data were then decomposed using Independent Component Analysis, and automated Independent Component (IC) selection was performed using IClabel for artifact IC rejection available in EEGLAB. If the generated probabilistic score of artifact components such as eye, heart, line noise and channel noise was more than $70 \%$, then that artifact component was rejected. One male participant data was excluded from the analysis due to very noisy data. Therefore, EEG analysis was conducted on a sample of 10 participants.

ERP analysis focused on those electrodes and time intervals that have been identified to be associated with image repetition in previous studies. These Regions of Interests (ROIs) are frontal (F8, F4, AF4, FP2, FP1, AF3, F3, F7), centroparietal (C3, Cz, C4, CP1, CP2, P3, Pz, P4), parietooccipital (PO3, $\mathrm{PO} 4, \mathrm{O} 2, \mathrm{Oz}, \mathrm{O} 1, \mathrm{Pz})$ and occipitotemporal (P8, T8, P4, PO3, O1, Oz, O2, PO4, P3, T7, P7) [11], [4], [12]. In order to analyse early window processing, negative peak ERP amplitude after visual stimulus onset was measured in the time window of $150-300 \mathrm{~ms}$ in all ROIs mentioned above. For LPP, positive peak amplitude was measured over all ROIs in the time window $300-600 \mathrm{~ms}$ after visual stimulus onset.

\section{RESULTS}

Significant main effects and interactions for ERPs were analysed in early (150-300ms) and late time windows (300$600 \mathrm{~ms}$ ) in parietal, frontal, central and occipitotemporal areas of the brain and the results are provided in this section. The pre-hoc non-parametric equivalent of the repeated measure analysis of variance (Friedman test) included the factor repetitions (apple, pizza and hammer images) and four 
regions. Post-hoc analysis with Wilcoxon signed-rank test was conducted using Bonferroni correction, resulting in a significance level set at $p<0.017$ i.e. $0.05 / 3$.

\section{A. Early ERP Interval (150-300ms)}

In early ERP interval, statistical significance can be seen at frontal, parietooccipital and occipitotemporal sites but not at centroparietal sites. A non-parametric test of differences among repeated measures in the frontal region was conducted and it showed statistical significance $\left(\chi^{2}(2)=47.74, \mathrm{p}=\right.$ 4.28e-11). Post-hoc analysis results showed that significant differences were found between pizza and apple $(Z=-3.91$, $\mathrm{p}=1.53 \mathrm{e}-05)$, between pizza and hammer $(Z=-6.85, \mathrm{p}=$ $1.18 \mathrm{e}-12)$, and between apple and hammer $(Z=-3.10, \mathrm{p}=$ $3.21 \mathrm{e}-04)$. In the parietooccipital region, there was significant difference between images $\left(\chi^{2}(2)=22.54, \mathrm{p}=1.27 \mathrm{e}-05\right)$, but although there was significant difference between pizza and other images (with apple $(Z=6.54, \mathrm{p}=9.85 \mathrm{e}-12)$ and with hammer $(Z=4.77, \mathrm{p}=3.04 \mathrm{e}-07))$, there was no significant difference between apple and hammer $(Z=-0.44, \mathrm{p}=0.22)$. Also, significant difference was found between images in the occipitotemporal region $\left(\chi^{2}(2)=27.87, \mathrm{p}=8.84 \mathrm{e}-07\right)$; in particular, between pizza and apple $(Z=6.31, \mathrm{p}=4.51 \mathrm{e}-$ $11)$, pizza and hammer $(Z=6.29, \mathrm{p}=5.15 \mathrm{e}-11)$, and apple and hammer $(Z=2.52, \mathrm{p}=1.9 \mathrm{e}-03)$.

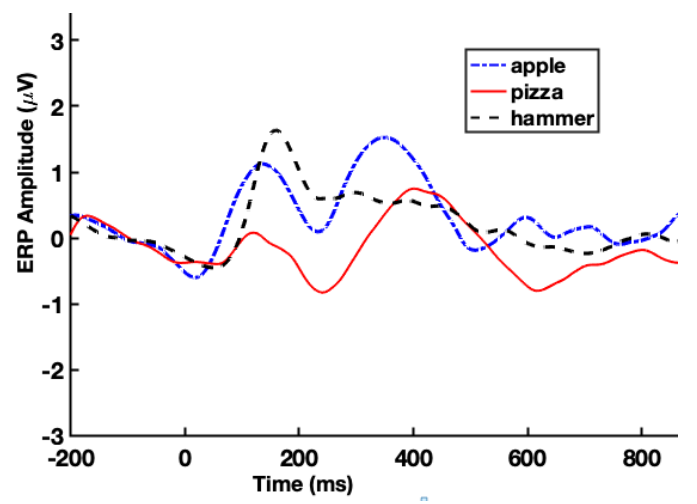

Fig. 2: Grand averaged ERP over frontal region for apple, pizza and hammer images

Fig. 2 illustrates the early window ERP waveforms for high caloric, low caloric and neutral images averaged across all participants. Early ERP shows increased negative amplitude for pizza more than apple and hammer, and among apple and hammer, apple shows higher negative amplitude.

\section{B. Late ERP Interval (300-600ms)}

In late ERP interval, statistical significance can be seen at centroparietal, parietooccipital and occipitotemporal sites but not at frontal sites. In the parietooccipital region, there was significant difference between images $\left(\chi^{2}(2)=51.51\right.$, $\mathrm{p}=6.53 \mathrm{e}-12)$. In particular, there was significant difference between pizza and apple $(Z=7.74, \mathrm{p}=1.63 \mathrm{e}-15)$, pizza and hammer $(Z=6.41, \mathrm{p}=2.49 \mathrm{e}-11)$, but no significance between apple and hammer $(Z=-0.27, \mathrm{p}=0.20)$. In the occipitotemporal region, there was significant difference between images $\left(\chi^{2}(2)=74.54, \mathrm{p}=6.50 \mathrm{e}-17\right)$. Post-hoc analysis showed that there was significant difference between pizza and apple $(Z=9.01, \mathrm{p}=3.35 \mathrm{e}-20)$, pizza and hammer $(Z=9.42, \mathrm{p}=6.9 \mathrm{e}-22)$, and apple and hammer $(Z=3.14$, $\mathrm{p}=2.79 \mathrm{e}-04)$. Over the centroparietal region, there was also significant difference between images $\left(\chi^{2}(2)=17.52, \mathrm{p}=\right.$ 1.57e-04). Significant differences were between pizza and apple $(Z=2.44, \mathrm{p}=2.43 \mathrm{e}-3)$, pizza and hammer $(Z=4.16, \mathrm{p}$ $=5.27 \mathrm{e}-06)$, and apple and hammer $(Z=2.64, \mathrm{p}=1.35 \mathrm{e}-03)$. Fig. 3 illustrates the ERP waveforms for high caloric, low

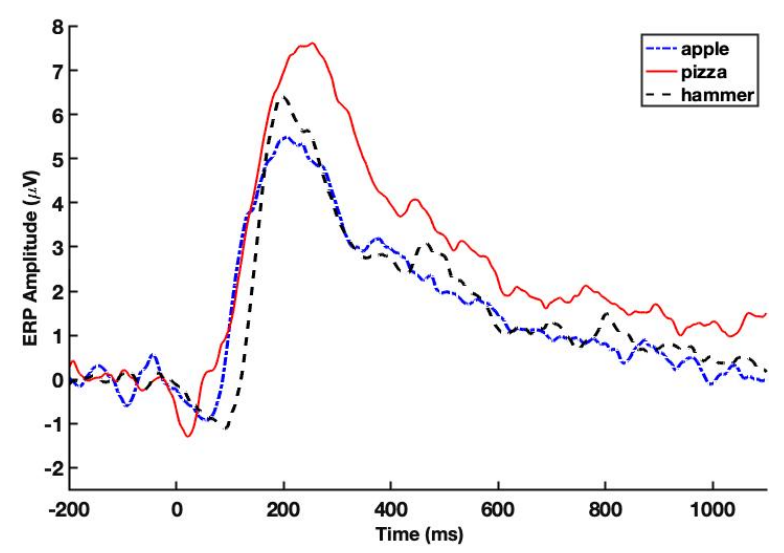

Fig. 3: Grand averaged ERP over occipitotemporal region for apple, pizza and hammer images

caloric and neutral images averaged across all participants over the late window. The amplitude of late ERP shows more positive motivational attention towards pizza than apple and hammer, but apple shows similar motivational attention to hammer.

\section{DISCUSSION}

Effects of repetition of the same food and non-food images on the modulation of early and late ERP windows were investigated in this study. This study mainly focuses on comparing the evoked potentials and behavioural response to the repeated presentation rather than on technical design challenges; for this, standard data processing methods have been utilised. The results show that larger amplitude was associated with food images than non-food image in the early and late ERP windows, especially for the high caloric pizza image. This indicates that these two ERP components, which are modulated by arousal, may reflect the same pattern of attention towards food and non-food image processing.

In studies investigating food processing via image viewing tasks, in the early ERP window, N2 is shown to be related to conflict response and cognitive inhibitory control [13]. Existing study shows increased frontal negativity (defined as "Anterior Negativity") found for varied chocolate images more than neutral images in binge eating individuals [4]. Asmaro et al. suggested that the declination in the frontal region of the brain might be related to top-down cognitive control mechanism towards the desire to consume chocolates in non-cravers [14]. 
However, with regard to the processing of food stimuli, in existing studies, higher anterior negative deflections in the N2 showed that higher amplitudes were related to increased reactivity to food stimuli [14]. Similar results can be observed in affective related studies as well, where emotion pictures show higher negativity than neutral pictures in the early ERP window [11]. In this study as well, similar results have been identified, with high caloric food showing increased negativity with respect to low caloric and non-food images when the same image was presented to participants repeatedly. This interaction effect might indicate that there was a relative increase in cognitive control in response to high caloric images more than other images. This shows that in the early ERP window, an individual's appetitive behaviour and motivational attention do not vary between identical or inter-mixed presentations identified in other studies [14], [4].

On the other hand, in late positive potential, over occipitotemporal channels, results highlight that food images, especially high caloric food image, continued to elicit larger positive potentials than neutral images. This suggests that engaging in food images sustain to draw attentional resources, despite the previous presentation. These results confirm the existing ERP studies where the repetition of variety of food images reflects high motivational significance, especially in high caloric food versus non-food images [3].

This is the first study to show differentiation in motivational attention when same food images (high caloric and low caloric food) and non-food images were presented repeatedly. Considering early and LPP results, this study finds that the repetitive exposure to the same food image continues to activate the neural circuits that influence appetitive or defensive motivation more than non-food images, especially for high caloric food. This interaction effect might indicate that, although the images were highly familiar to participants because of repetition of the same image, the initial encoding and the attention allocation processes were obligatory for each image identification and continued to be modulated by hedonic valence. Also, in the early ERP window, results obtained in this study (high negativity for food images) reflect similar results from affective studies where emotional images show higher negativity than neutral images [11]. This suggests that emotion towards food images plays a more significant role in modulating the LPP window than towards non-food images, with high emotion been associated more with high caloric food than low caloric and non-food images.

\section{Conclusion}

Effects of modulation of early and late ERP window during repeated exposure of the same food and non-food images was investigated in this study. Data suggests that high caloric food in both early and late ERP windows continued to show greater attention than low caloric and non-food image. In the early ERP window, participants show increased negative amplitude for high caloric images more than other images in the frontal region. This negative deflection might be related to top-down cognitive control mechanisms over desire to consume high caloric food. On the other hand, in the late ERP window, participants show sustained positive amplitude for high caloric images more than low caloric or non-food images in the occipitotemporal region. This shows that high caloric food continues to draw attention more than other images. In conclusion, the early and late ERP component results of this study can be compared with other studies [3], [4] where a variety of images are used and suggest that the effect of repetition of food and nonfood images can be obtained by the presentation of a single image repeatedly rather than using a variety of images. This might be helpful in designing future repetitive studies to use single visual food image instead of inter-mixed images to get the same effect.

As the study mainly focused on investigating the effects of repetition of same energy valued images, future work will include an in-depth analysis of repetition of the same image between high and low BMI groups and between types of eaters (external, restrained, etc.). Furthermore, subjective craving intensity data will be studied to determine any correlation between subjective and neurophysiological data.

\section{REFERENCES}

[1] "World health organization," World Health Organization, Apr 16, 2021. [Online]. Available: https://www.who.int/health-topics/obesity

[2] I. Krajbich, C. Armel, and A. Rangel, "Visual fixations and the computation and comparison of value in simple choice," Nat Neurosci, vol. 13, pp. 1292-1298, 2010.

[3] C. V. Lietti, M. M. Murray, J. Hudry, J. le Coutre, and U. Toepel, "The role of energetic value in dynamic brain response adaptation during repeated food image viewing," Appetite, vol. 58, pp. 11-18, 2012.

[4] I. Wolz, A. Sauvaget, R. Granero, G. Mestre-Bach, M. Baño, V. Martín-Romera, M. Veciana De Las Heras, S. Jiménez-Murcia, A. Jansen, A. Roefs, and F. Fernández-Aranda, "Subjective craving and event-related brain response to olfactory and visual chocolate cues in binge-eating and healthy individuals," Scientific Reports, vol. 7, 2017.

[5] M. M. Bradley, "Natural selective attention: Orienting and emotion," Psychophysiology, vol. 46, no. 1, pp. 1-11, 2009.

[6] M. De Lucia, L. Cocchi, R. Martuzzi, R. A. Meuli, S. Clarke, and M. M. Murray, "Perceptual and Semantic Contributions to Repetition Priming of Environmental Sounds," Cerebral Cortex, vol. 20, no. 7, pp. 1676-1684, 2010.

[7] L. H. Epstein, J. L. Temple, and M. E. Bouton, "Human Food IntakeHabituation," Psychol Rev, vol. 116, no. 2, pp. 384-407, 2009.

[8] L. H. Epstein, J. L. Robinson, J. L. Temple, J. N. Roemmich, A. L. Marusewski, and R. L. Nadbrzuch, "Variety influences habituation of motivated behavior for food and energy intake in children," Am J Clin Nutr, vol. 89, no. 3, pp. 746-754, 2009.

[9] M. W. Geisler and J. Polich, "P300, Food Consumption, and Memory Performance," Psychophysiology, vol. 29, no. 1, pp. 76-85, 1992.

[10] N. Bigdely-Shamlo, T. Mullen, C. Kothe, K. M. Su, and K. A. Robbins, "The PREP pipeline: Standardized preprocessing for largescale EEG analysis," Front Neuroinform, vol. 9, pp. 1-19, 2015.

[11] M. Codispoti, V. Ferrari, and M. M. Bradley, "Repetition and eventrelated potentials: Distinguishing early and late processes in affective picture perception," Journal of Cognitive Neuroscience, vol. 19, no. 4, pp. 577-586, 2007.

[12] V. Ferrari, M. M. Bradley, M. Codispoti, and P. J. Lang, "Repetitive exposure: Brain and reflex measures of emotion and attention," Psychophysiology, vol. 48, no. 4, pp. 515-522, 2011.

[13] T. E. Baker and C. B. Holroyd, "Dissociated roles of the anterior cingulate cortex in reward and conflict processing as revealed by the feedback error-related negativity and N200," Biological Psychology, vol. 87, no. 1, pp. 25-34, 2011.

[14] D. Asmaro, F. Jaspers-Fayer, V. Sramko, I. Taake, P. Carolan, and M. Liotti, "Spatiotemporal dynamics of the hedonic processing of chocolate images in individuals with and without trait chocolate craving," Appetite, vol. 58, no. 3, pp. 790-799, 2012. 